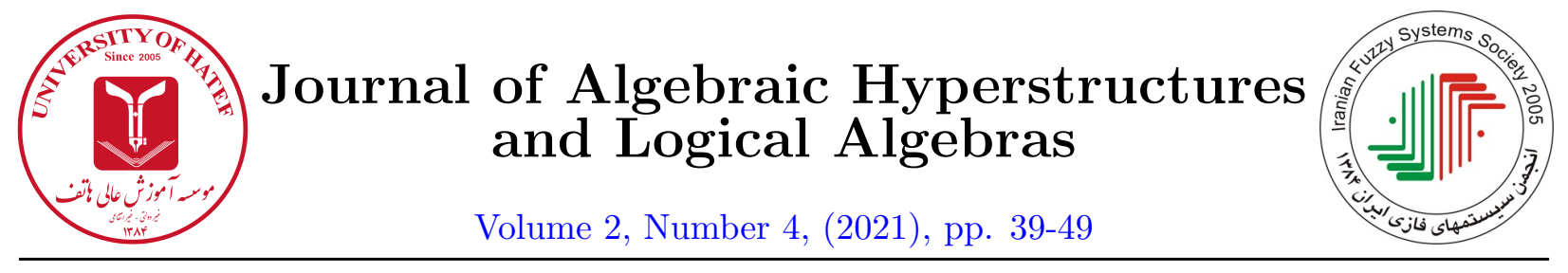

\title{
On autosolvable and autonilpotent polygroups
}

\author{
A. Mosayebi Dorcheh ${ }^{1}$ \\ ${ }^{1}$ Department of Mathematies, Payame Noor University (PNU), Iran \\ alimosayebi@pnu.ac.ir
}

\begin{abstract}
Polygroups are another important class of hypergroups. The importance of polygroups is their connection to graphs, relations and Boolean algebras. In this paper, we study notions of autosolvable and autonilpotent polygroups by using the heart of a polygroup. This study introduces the concept of autosolvable and autonilpotent polygroups with respect to the automorphism of polygroups. We also prove that autonilpotent polygroups are autosolvable.
\end{abstract}

\section{Article Information}

Corresponding Author:

A. Mosayebi Dorcheh;

Received: September 2021;

Revised: November 2021;

Accepted: November 2021;

Paper type: Original.

\section{Keywords:}

Automorphism, autosolvable polygroup, autocommutator.

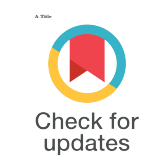

\section{Introduction}

The hyperstructure theory was firstly introduced, by F. Marty at the 8th congress of Scandinavian Mathematicians in 1934. Marty introduced the concept of hypergroups as a generalization of groups and used it in different contexts like algebraic functions, rational fractions and non-commutative groups. In classical algebraic structures, the synthetic result of two elements is an element, while in the hyper algebraic system, the synthetic result of two elements is a set of elements, therefore it can be said that the notion of hyperstructures is a generalization of classical algebraic structures, from this point of view. Hyperstructures have many applications to several sectors of both pure and applied sciences as geometry, graphs and hypergraphs, fuzzy sets and rough sets, automata, cryptography, codes, relation algebras, C-algebras, artificial intelligence, probabilities, chemistry, physics, especially in atomic physics and in harmonic analysis. In this paper, we introduce a type of automorphism using the automorphism of any hypergroup and invariant congruence relation on a hypergroup and express a congruence relation between automorphisms and study its properties.

https://doi.org/10.52547/HATEF.JAHLA.2.4.4 
Finally, we introduce the autosolvable and autonilpotent polygroup using the concept of autocommutators and study relationship between autosolvable and autonilpotent polygroups.

\section{Preliminaries}

Let $H$ be a non-empty set and $P^{*}(H)$ be the set of all non-empty subsets of $H$. Let - be a hyperoperation on $H$, that is, - is a map from $H \times H$ into $P^{*}(H)$ and structure $(H, \cdot)$ is called a hypergroupoid. For any two non-empty subsets $A$ and $B$ of $H$ and $x \in H$, we define $A \cdot B=$ $\bigcup_{a \in A, b \in B} a \cdot b, A \cdot x=A \cdot\{x\}, x \cdot B=\{x\} \cdot B$.

A hypergroupoid $(H, \cdot)$ is called a semihypergroup if for all $a, b, c$ of $H$ we have $(a \cdot b) \cdot c=a \cdot(b \cdot c)$. A semihypergroup is a hypergroup if $a \cdot H=H \cdot a=H$ for all $a \in H$. Let $\left(H_{1}, \bullet_{1}\right)$ and $\left(H_{2}, \bullet_{2}\right)$ be hypergroups. Define a hyperoperation $\bullet$ on $H_{1} \times H_{2}$ by $(x, y) \bullet(z, w)=\left(x \bullet{ }_{1} z, y \bullet \bullet_{2} w\right)$. Then $\left(H_{1} \times H_{2}, \bullet\right)$ is a hypergroup. The map $f: H_{1} \rightarrow H_{2}$ is called a homomorphism (or inclusion homomorphism) of hypergroups if for all $a, b \in H_{1}$, we have $f(a \cdot b)=f(a) \cdot f(b)$ ( or $f(a \cdot b) \subseteq f(a) \cdot f(b))$. A homomorphism $f$ is called an isomorphism if $f$ is a one to one and onto map. If $H$ is a hypergroup, an (inclusion) automorphisms of $H$ is an (inclusion) isomorphism from $H$ to $H$. The set of (inclusion) automorphisms of $H$ denoted by $A u t(H)(A u t I(H))$. In every hypergroup $H$, a commutator of $x, y \in H$ is denoted by $[x, y]=\{h \in H \mid x \cdot y \cap h \cdot y \cdot x \neq \emptyset\}$ (See [四]). Let $x \in H$ and $\alpha \in \operatorname{Aut}(H)$. Define $[x, \alpha]=\{h \in H \mid x \in h \cdot \alpha(x)\}$ and will call an autocommutator of $x$ and $\alpha$. Inductively, for all $\alpha_{1}, \alpha_{2}, \cdots \alpha_{n} \in \operatorname{Aut}(H),\left[x, \alpha_{1}, \alpha_{2}, \cdots \alpha_{n}\right]=\left[\left[x, \alpha_{1}, \alpha_{2}, \cdots \alpha_{n-1}\right], \alpha_{n}\right]$, where for any two non-empty subsets $X \subseteq H$ and $A \subseteq A u t(H)$, we define $[X, \alpha]=\bigcup_{x \in X}[x, \alpha]$ and $[X, A]=\bigcup_{\substack{x \in X \\ \alpha \in A}}[x, \alpha]$ (See $\left.[8]\right)$. Let $(H, \cdot)$ be a hypergroup and $R$ be an equivalence relation on $H$. Letting $H / R=\{\bar{x} \mid x \in H\}$, be the set of all equivalence classes of $H$ with respect $R$. Define a hyperoperation $\circ$ as follows: $\bar{x} \circ \bar{y}=\{\bar{z} \mid z \in \bar{x} \cdot \bar{y}\}$. In [10]] it was proved that $(H / R, \circ)$ is a group if and if only $R$ is a strongly regular equivalence relation. A (strongly) regular equivalence relation we call (strongly) congruence relation.

Let $H$ be a hypergroup and $R$ be a relation on $H$. Then $R$ is called an invariant relation, if for all $\alpha \in \operatorname{Aut}(H)$, we have $\alpha(R) \subseteq R$, where $\alpha(R)=\{\alpha(x, y):=(\alpha(x), \alpha(y)) \mid(x, y) \in R\}$. A semihypergroup $(P, \cdot)$ is called a polygroup, provided that $(i)$ it has a scaler identity e, that is, $e \cdot x=x \cdot e=\{x\}$, for every $x \in P,($ ii $) x \in y \cdot z$ implies $y \in x \cdot z^{-1}$ and $z \in y^{-1} \cdot x$, where ${ }^{-1}$ is an unitary operation on $H$. A non-empty subset $K$ of $P$ is said to be a subpolygroup of $P$, if for any $x, y \in K, x \cdot y^{-1} \subseteq K$ and is denoted by $K \leq P$. A subpolygroup $K$ of $P$ is called a normal subpolygroup, if for any $x \in P, x^{-1} \cdot K \cdot x \subseteq K$ and is denoted by $K \triangleleft P$. A subpolygroup $K$ of $P$ is said to be characteristic in $P$ if $\alpha(K) \subseteq K$ for all $\alpha \in A u t(P)$, and we denote it by $K \leq \leq_{c} P$. Notice that if $K$ is characteristic in $P$ and $\alpha \in A u t(P)$, then $\alpha(K)=K$. There are several commonly used notations for the image of $(x, y)$ under a binary hyperoperation: $x y$ (multiplicative notation), $x \circ y, x * y$, etc. For convenience we shall generally use the multiplicative notation throughout this paper and refer to $x y$ as the product of $x$ and $y$.

Suppose that $A$ and $B$ are two polygroups and $A \cap B=\{e\}$. Then $(A[B], *)$ is a polygroup (See [4]) as follows: $x * e=e * x=x$ for all $x \in A \cup B$, and for all $x, y \in A \cup B-\{e\}$,

$$
x * y= \begin{cases}x y & \text { if } x, y \in A \\ x & \text { if } x \in B, y \in A \\ y & \text { if } x \in A, y \in B \\ x y & \text { if } x, y \in B, y \neq x^{-1} \\ x y \cup A & \text { if } x^{-1}=y \in B\end{cases}
$$


Let $X$ be a non-empty subset of a polygroup $P$ define the subpolygroup generated by $X,\langle X\rangle$ to be the intersection of all subpolygroups of $P$ which contain $X$. Let $\mathcal{U}$ denote the set of all finite product of elements of $P$. Then $\omega_{P}=\{x \in P \mid \exists u \in \mathcal{U}$ s.t $\quad e, x \in u\}$ is called heart of $P$. Polygroup $P$ is said to be nilpotent if $l_{n}(P) \subseteq \omega_{P}$, for some integer $n$, where $l_{0}(P)=P$ and

$$
l_{n+1}(P)=\left\langle\left\{h \in[x, y] \mid x \in l_{n}(P), y \in P\right\}\right\rangle .
$$

A polygroup $P$ is called solvable if $P^{(n)}=\omega_{P}$, for some $n \in \mathbb{N}$, where $P^{(1)}=P^{\prime}=\langle[P, P]\rangle$, and $P^{(n+1)}=\left(P^{(n)}\right)^{\prime}$.

Define a relation $\beta$ on $H$ by $a \beta b \Longleftrightarrow \exists u \in \mathcal{U}(H)$ such that $\{a, b\} \subseteq u$. If $\beta^{*}$ is the transitive closure of $\beta$. In [4] it was rewrote the definition of $\beta^{*}$ on $H$ as follows:

$$
a \beta^{*} b \Longleftrightarrow \exists z_{1}=a, z_{2}, \ldots, z_{n+1}=b \in H, u_{1}, u_{2} . ., u_{n} \in \mathcal{U} \text { s.t }\left\{z_{i}, z_{i+1}\right\} \subseteq u_{i}, \forall 1 \leq i \leq n .
$$

Also Freni, introduced a strongly regular relation $\gamma$ on a hypergroup $H$ as follows:

$$
\Delta=\gamma_{1}=\{(x, x) \mid x \in H\},
$$

and for all $n \geq 2,(x, y) \in \gamma_{n}$ if and only if there exist $z_{1}, z_{2}, \ldots, z_{n} \in H, \sigma \in S_{n}$ such that $x \in \prod_{i=1}^{n} z_{i}$, $y \in \prod_{i=1}^{n} z_{\sigma(i)}=u_{\sigma}$ and $\gamma=\bigcup_{n \geq 1} \gamma_{n}$, where $S_{n}$ is the symmetric group of degree $n$, in addition it was

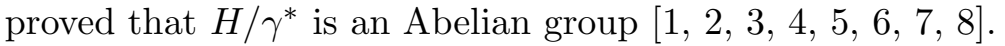

B. Davvaz et al, introduced the relation $\nu_{n}=\bigcup_{m \geq 1} \nu_{m, n}$, where $\nu_{1, n}=\gamma_{1}$ and for every $m>1$, $\nu_{m, n}$ is defined by, $(a, b) \in \nu_{m, n} \Leftrightarrow \exists u=\prod_{i=1}^{m} z_{i} \in \mathcal{U}, \exists \sigma \in S_{m}$ such that $\sigma(i)=i$ if $z_{i} \notin L_{n}(H)$ and $a \in u, b \in u_{\sigma}$, in addition it was proved that $H / \nu_{n}^{*}$ is a nilpotent group [I]. Also $\tau_{n}=\bigcup_{m \geq 1} \tau_{m, n}$, where $\tau_{1, n}=\{(x, x) \mid x \in H\}$ and for every $m>1, \tau_{m, n}$ is defined by,

$$
(a, b) \in \tau_{m, n} \Leftrightarrow \exists u=\prod_{i=1}^{m} z_{i}, \exists \sigma \in S_{m}: \sigma(i)=i \text { if } z_{i} \notin \Gamma_{n}(H) \text { and } a \in u, b \in u_{\sigma},
$$

in addition it was proved that $H / \tau_{n}^{*}$ is a solvable group [2]. Also, A. Mosayebi Dorcheh introduced a strongly regular relation $R_{K}$ on a hypergroup $H$ and it was proved that $H / R_{K}^{*}$ is a $k$-nilpotent group [7].

In [8], Mosayebi et al, introduced the notion of auto-Engel polygroup and investigated some properties of it. Moreover they defined the concept of characteristic sets in hypergroups and gave some related properties. In [9], Moghaddam and Parvaneh defined the concept of autonilpotent and autosolvable groups (by definition $[x, \alpha]=x^{-1} \alpha(x)$ ). Now, in this paper we define the notion of autonilpotent and autosolvable (according to the definition $[x, \alpha]=x \alpha\left(x^{-1}\right)$ ) and investigate some properties of them.

\section{Relation-equality in automorphisms}

In this section of the article, we introduce specific automorphisms using automorphisms on hypergroup and invariant relation. 
Lemma 3.1. Let $(P, \cdot, e)$ be a polygroup and $\alpha \in A u t I(P)$ and $x \in P$. Then

(i) $\alpha(e)=e$,

(ii) $\alpha\left(x^{-1}\right)=\alpha(x)^{-1}$,

(iii) $[x, \alpha]=x \alpha\left(x^{-1}\right),[\alpha, x]:=[x, \alpha]^{-1}=\alpha(x) x^{-1}=\left[\alpha(x), \alpha^{-1}\right]$.

Proof. (i) Let $x \in P$ and $\alpha(x)=e$. Then we have

$$
\{e\}=\{\alpha(x)\}=\alpha(e \cdot x) \subseteq \alpha(e) \cdot \alpha(x)=\alpha(e) \cdot e=\{\alpha(e)\} .
$$

This yields $\alpha(e)=e$.

(ii) Let $a \in P$. Then we have $e \in a a^{-1}$ and thus $\alpha(e) \in \alpha(a) \alpha\left(a^{-1}\right)$. Now, we conclude that $\alpha(a)^{-1}=\alpha\left(a^{-1}\right)$.

(iii) $[\alpha, x]=[x, \alpha]^{-1}=\left(x \alpha\left(x^{-1}\right)\right)^{-1}=\alpha(x) x^{-1}=\left[\alpha(x), \alpha^{-1}\right]$.

Lemma 3.2. Let $H$ be a hypergroup and $R$ be an invariant strongly congruence relation on $H$, $\alpha \in \operatorname{Aut}(H)$. Then there is an automorphism $\bar{\alpha}: H / R \rightarrow H / R$ such that $\bar{\alpha} \pi=\pi \alpha$, that is to say, such that the diagram commutes, where $\bar{\alpha}(\bar{x})=\overline{\alpha(x)}, x \in H$.

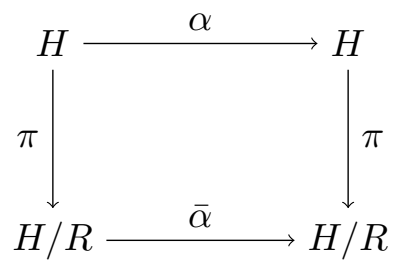

Figure 1:

Proof. Let $x, y \in H$ and $\bar{x}=\bar{y}$. Then

$$
(x, y) \in R \Rightarrow(\alpha(x), \alpha(y)) \in R \Rightarrow \bar{\alpha}(\bar{x})=\overline{\alpha(x)}=\overline{\alpha(y)}=\bar{\alpha}(\bar{y}) .
$$

Now, let $z \in x y$. Then, $\bar{\alpha}(\bar{x} \bar{y})=\bar{\alpha}(\bar{z})=\overline{\alpha(z)}=\overline{\alpha(x)} \overline{\alpha(y)}$, and hence $\alpha$ is a homomorphism. It is easy to see that $\alpha$ is an isomorphism, such that for all $x \in H, \bar{\alpha} \pi(x)=\pi(\alpha(x))$.

Corollary 3.3. Let $H$ be a hypergroup and $R$ be an invariant strongly congruence relation on $H$. Then,

(i) If $\alpha_{1}, \alpha_{2} \in \operatorname{Aut}(H)$, then $\overline{\alpha_{1}} \overline{\alpha_{2}}=\overline{\alpha_{1} \alpha_{2}}$,

(ii) For $\alpha \in \operatorname{Aut}(H), \bar{\alpha}^{-1}=\overline{\alpha^{-1}}$,

(iii) $\overline{\operatorname{Aut}(H)}=\{\bar{\alpha} \mid \alpha \in \operatorname{Aut}(H)\} \leq \operatorname{Aut}(H / R)$.

Proof. (i) Let $x \in H$. Then $\overline{\alpha_{1} \alpha_{2}}(\bar{x})=\overline{\alpha_{1} \alpha_{2}(x)}=\overline{\alpha_{1}}\left(\overline{\alpha_{2}(x)}\right)=\overline{\alpha_{1}}\left(\overline{\alpha_{2}}(\bar{x})\right)=\overline{\alpha_{1}} \overline{\alpha_{2}}(\bar{x})$.

(ii) By $(i)$, we have $\bar{\alpha} \overline{\alpha^{-1}}=\overline{\alpha \alpha^{-1}}=\bar{I}$, where $I$ is the identity of the group $\operatorname{Aut}(H)$. 
(iii) Let $\overline{\alpha_{1}}, \overline{\alpha_{2}} \in \overline{\operatorname{Aut}(H)}$. Then $\overline{\alpha_{1}}{\overline{\alpha_{2}}}^{-1}=\overline{\alpha_{1}} \overline{\alpha_{2}^{-1}}=\overline{\alpha_{1} \alpha_{2}^{-1}} \in \overline{\operatorname{Aut}(H)}$.

Definition 3.4. Let $H$ be a hypergroup and $R$ be an invariant (strongly) congruence relation on $H$. Then the two automorphisms $\alpha_{1}$ and $\alpha_{2}$ are called $R$-equality if $\left(\alpha_{1}(x), \alpha_{2}(x)\right) \in R$, for all $x$ of $H$. In this case we write $\alpha_{1}={ }_{R} \alpha_{2}$ and it is easy to see that $\overline{\alpha_{1}}=\overline{\alpha_{2}}$. In the special case $=_{\Delta}$ is the equality of automorphisms.

Example 3.5. Let $G=S_{3}=\left\{e, a, a^{2}, b, a b, a^{2} b\right\}$ and $\{e, c\}$ be a cyclic group and $P=\{e, c\}[G]$. Then $\left(a a^{2}\right)=_{\gamma} I$ and so $\overline{\left(a a^{2}\right)}=\bar{I}$, but $\left(a a^{2}\right) \neq_{\beta} I$, where $\beta$ and $\gamma$ are the relations defined in the previous section.

Corollary 3.6. Let $\alpha_{1}, \alpha_{2}, \alpha \in$ Aut $(H)$. Then

(i) If $\alpha_{1}={ }_{R} \alpha_{2}$, then $\alpha_{1}^{-1}={ }_{R} \alpha_{2}^{-1}$,

(ii) If $\alpha_{1} \alpha_{2}={ }_{R} I$, then $\alpha_{1}^{-1}={ }_{R} \alpha_{2}$,

(iii) If $\alpha \alpha_{1}={ }_{R} \alpha \alpha_{2}$, then $\alpha_{1}={ }_{R} \alpha_{2}$,

(iv) If $\alpha_{1}={ }_{R} \alpha_{2}$ and $R \subseteq S$ and $S$ is an invariant (strongly) congruence relation, then $\alpha_{1}={ }_{S} \alpha_{2}$.

Proof. (i) Suppose that $\alpha_{1}={ }_{R} \alpha_{2}$. Then, we have $\overline{\alpha_{1}}=\overline{\alpha_{2}}$ and hence ${\overline{\alpha_{1}}}^{-1}={\overline{\alpha_{2}}}^{-1}$. Therefore, by definition $\alpha_{1}^{-1}={ }_{R} \alpha_{2}^{-1}$.

(ii) We have $\overline{\alpha_{1}} \overline{\alpha_{2}}=\bar{I}$ and so $\overline{\alpha_{1}}{ }^{-1}=\overline{\alpha_{2}}$. It follows that $\alpha_{1}^{-1}={ }_{R} \alpha_{2}$.

(iii) By definition the proof is clear.

(iv) Let $\alpha_{1}={ }_{R} \alpha_{2}$ and $R \subseteq S$. Then for every $x \in H$ we have $\left(\alpha_{1}(x), \alpha_{2}(x)\right) \in R$ and so $\left(\alpha_{1}(x), \alpha_{2}(x)\right) \in S$, for all $x \in H$ and thus $\alpha_{1}={ }_{S} \alpha_{2}$.

\section{Auto- $C$ class polygroups}

In this section our main purpose is to explore the structure of autocommutator, we investigate the concept of autosolvable polygroups and we give some results in this respect. Finally, we discuss on autonilpotent.

Definition 4.1. Let $P$ be a polygroup. Define

$$
A^{(1)}(P)=A(P)=\langle[P, A u t(P)]\rangle=\langle h \in[x, \alpha] \mid x \in P, \alpha \in \operatorname{Aut}(P)\rangle,
$$

and for every $n \in \mathbb{N}, A^{(n+1)}(P)=A\left(A^{(n)}(P)\right)=\left\langle\left[A^{(n)}(P)\right.\right.$, Aut $\left.\left.\left(A^{(n)}(P)\right)\right]\right\rangle$. Then $P$ is called an autosolvable polygroup of class at most $n$, if $A^{(n)}(P) \subseteq \omega_{P}$. Clearly, if $P=\omega_{P}$, then $P$ is an autosolvable polygroup. 
Example 4.2. (i) Let $P=\{1,2,3,4,5,6,7\}$. Then $(P, \cdot, 1)$ is a solvable polygroup as follows:

\begin{tabular}{l|lllllll}
$\cdot$ & 1 & 2 & 3 & 4 & 5 & 6 & 7 \\
\hline 1 & 1 & 2 & 3 & 4 & 5 & 6 & 7 \\
2 & 2 & $\{1,2\}$ & 3 & 4 & 5 & 6 & 7 \\
3 & 3 & 3 & $\{1,2\}$ & 7 & 6 & 5 & 4 \\
4 & 4 & 4 & 6 & $\{1,2\}$ & 7 & 3 & 5 \\
5 & 5 & 5 & 7 & 6 & $\{1,2\}$ & 4 & 3 \\
6 & 6 & 6 & 4 & 5 & 3 & 7 & $\{1,2\}$ \\
7 & 7 & 7 & 5 & 3 & 4 & $\{1,2\}$ & 6
\end{tabular}

(See [4] $)$. Since $\omega_{P}=\{1,2\}$ and $\operatorname{Aut}(P) \cong \operatorname{Aut}\left(S_{3}\right) \cong S_{3}$, we get $A(P)=\{1,2,6,7\}$ and therefore Aut $(A(P))=\{I,(6 \quad 7)\}$ and so for $n \geq 3, A^{(n)}(P)=\{1,2,6,7\}$. Thus, $P$ is a solvable polygroup which is not an autosolvable polygroup.

(ii) We consider $P=\{e, a, b\}$ whose table is given below:

\begin{tabular}{l|lll}
$\cdot$ & $e$ & $a$ & $b$ \\
\hline$e$ & $e$ & $a$ & $b$ \\
$a$ & $a$ & $\{e, b\}$ & $\{a, b\}$ \\
$b$ & $b$ & $\{a, b\}$ & $\{e, a\}$
\end{tabular}

Then, $\operatorname{Aut}(P)=\{I,(a b)\}$, and thus for all $n \geq 1, A^{(n)}(P)=P$. Therefore $P$ is not an autosolvable polygroup.

Example 4.3. Let $P=\{1,2,3,4,5,6,7,8,9\}$. Then $(P, \cdot, 1)$ is a polygroup as follows:

\begin{tabular}{l|lllllllll}
$\cdot$ & 1 & 2 & 3 & 4 & 5 & 6 & 7 & 8 & 9 \\
\hline 1 & 1 & 2 & 3 & 4 & 5 & 6 & 7 & 8 & 9 \\
2 & 2 & $\{1,2\}$ & 3 & 4 & 5 & 6 & 7 & 8 & 9 \\
3 & 3 & 3 & $\{1,2\}$ & 9 & 8 & 7 & 6 & 5 & 4 \\
4 & 4 & 4 & 7 & $\{1,2\}$ & 9 & 8 & 3 & 6 & 5 \\
5 & 5 & 5 & 8 & 7 & $\{1,2\}$ & 9 & 4 & 3 & 6 \\
6 & 6 & 6 & 9 & 8 & 7 & $\{1,2\}$ & 5 & 4 & 3 \\
7 & 7 & 7 & 4 & 5 & 6 & 3 & 8 & 9 & $\{1,2\}$ \\
8 & 8 & 8 & 5 & 6 & 3 & 4 & 9 & $\{1,2\}$ & 7 \\
9 & 9 & 9 & 6 & 3 & 4 & 5 & $\{1,2\}$ & 7 & 8
\end{tabular}

It is easy to see that $A u t(P) \cong A u t\left(D_{8}\right)$. Now, if

$$
D_{8}=\left\{e=1, a=7, a^{2}=8, a^{3}=9, b=3, a b=4, a^{2} b=5, a^{3} b=6,\right\},
$$

then we get $P / \beta \cong D_{8}$ and $A u t\left(D_{8}\right)=\left\{\alpha: D_{8} \rightarrow D_{8} \mid \alpha(a)=a^{i}, \alpha(b)=a^{j} b\right.$, where $i \in\{1,3\}, j \in$ $\{0,1,2,3\}\}$ and thus

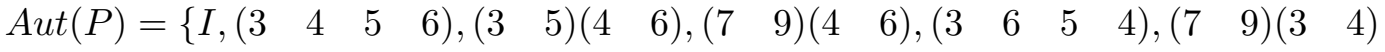

$$
\begin{aligned}
& \left.\left(\begin{array}{ll}
5 & 6
\end{array}\right),\left(\begin{array}{ll}
7 & 9
\end{array}\right)(3 \quad 5),\left(\begin{array}{lll}
7 & 9
\end{array}\right)\left(\begin{array}{ll}
3 & 6
\end{array}\right)\left(\begin{array}{ll}
4 & 5
\end{array}\right)\right\}
\end{aligned}
$$

It follows $A^{(1)}(P)=\{1,2,7,8,9\}$ and $\left.A u t(A(P))=\left\{\begin{array}{ll}I,(7 & 9\end{array}\right)\right\}$. Therefore, for $n \geq 3$ we have $A^{(n)}(P)=\{1,2\}=\omega_{P}$ and so $P$ is an autosolvable polygroup. 
Corollary 4.4. Let $P$ be a polygroup and for $n \in \mathbb{N}, \operatorname{Aut}\left(A^{(n)}(P)\right)=\{I\}$. Then $P$ is an autosolvable polygroup.

If $\operatorname{Aut}(P)=\{I\}$ obviously we have Aut $\left(A^{(n)}(P)\right)=\{I\}$ for every $n \in \mathbb{N}$.

Example 4.5. Let $P=\{e, a, b, c\}$. Then $(P, \cdot)$ is a polygroup as follows:

\begin{tabular}{l|llll}
$\cdot$ & $e$ & $a$ & $b$ & $c$ \\
\hline$e$ & $e$ & $a$ & $b$ & $c$ \\
$a$ & $a$ & $a$ & $P$ & $c$ \\
$b$ & $b$ & $\{e, a, b\}$ & $b$ & $\{b, c\}$ \\
$c$ & $c$ & $\{a, c\}$ & $c$ & $P$
\end{tabular}

(See [4]). Since Aut $(P)=\{I\}$, we get that $P$ is an autosolvable polygroup.

Corollary 4.6. Let $(P, \cdot, e)$ be a polygroup. Then,

(i) For all $n \in \mathbb{N}, e \in A^{(n)}(P)$,

(ii) For all $n \in \mathbb{N}, A^{(n+1)}(P) \subseteq A^{(n)}(P)$,

(iii) If $P$ is an autosolvable polygroup of class $n$, then $P$ is an autosolvable polygroup of class $n+1$.

(iv) For all $n \in \mathbb{N}$,

$$
A^{(n)}(P)=\left\langle\left[x, \alpha_{1}, \ldots, \alpha_{n}\right] \mid x \in P, \alpha_{i} \in \operatorname{Aut}\left(A^{(i-1)}(P)\right), i=1, \ldots, n\right\rangle,
$$

where $A^{(0)}(P)=P$.

Proof. (i) Obviously for all $n$, we have $I \in A$ ut $\left(A^{(n)}(P)\right)$ and for all $x \in P, e \in x \cdot x^{-1}=[x, I]$. Thus $e \in A^{(n)}(P)$.

(ii) By induction the proof is obtained.

(iii) By item (ii) it is clear.

(iv) It is an immediate consequence of definition.

Corollary 4.7. A group $G$ is autosolvable of class $n$ if and only if $A^{(n)}(G)=\{e\}$.

Proof. By Corollary (4.6) we have $e \in A^{(n)}(G)$, but $\omega_{G}=\{e\}$ and thus $A^{(n)}(G) \subseteq\{e\}$ if and only if $A^{(n)}(G)=\{e\}$.

Theorem 4.8. Let $G$ be an autosolvable group. Then $G$ is a solvable group.

Proof. We prove $G^{(n)} \subseteq A^{(n)}(G)$ by induction on $n$, where $G^{(1)}=G^{\prime}=\langle[G, G]\rangle$ and for every $k \geq 1$,

$$
G^{(k+1)}=\left\langle\left[G^{(k)}, G^{(k)}\right]\right\rangle .
$$

Now, suppose that $h=[x, y]$, where $x, y \in G^{(n)}$ so by hypothesis of induction $x, y \in A^{(n)}(G)$. Now, if for every $a \in G, \phi_{y}(a)=y a y^{-1}$ is the inner automorphism group of $G$, then, we have

$$
h=[x, y]=x y x^{-1} y^{-1}=\left[x, \phi_{y}\right] \in A^{(n+1)}(G) .
$$

Finally let $G$ be an autosolvable group of class $n$, then by Corollary (4.7) we get $G^{(n)}=\{e\}$. 
Example 4.9. (i) Let $G=\mathbb{Z}_{6}$. Since for $n \geq 1, A^{(n)}(G)=\{\overline{0}, \overline{2}, \overline{4}\}$, and Aut $\left(A^{(n)}(G)\right)=$ $\{I,(\overline{2} \overline{4})\}$, we have $A^{(n)}(G) \neq\{\overline{0}\}$, and so $G$ is a solvable group which is not an autosolvable group. Thus the converse of Theorem 4.8 is not neccessarily true.

(ii) Let $G$ be a group and $\operatorname{Aut}(G)=\operatorname{Inn}(G)$. Then $A^{(n)}(G)=G^{(n)}$. In this case, we have the property of solvable groups.

Theorem 4.10. Let $P$ be a polygroup and for all $n \in \mathbb{N}, \operatorname{Aut}\left(A^{(n)}(\bar{P})\right) \subseteq \overline{\operatorname{Aut}\left(A^{(n)}(P)\right)}$. Then

(i) $P$ is an autosolvable polygroup if and only if $\bar{P}$ is an autosolvable group, where $\bar{P}=P / \beta$.

(ii) If $P$ is an autosolvable polygroup, then $P$ is solvable.

Proof. (i) First, we prove that $A^{(n)}(\bar{P})=\overline{A^{(n)}(P)}$, for all $n$ by induction on $n$. Let $\bar{a} \in$ $A^{(n+1)}(\bar{P})$, without losing generality suppose that $\bar{a}=[\bar{x}, \alpha]$, where $\bar{x} \in A^{(n)}(\bar{P})$ and $\alpha \in \operatorname{Aut}\left(A^{(n)}(\bar{P})\right)$. Thus there exists $\alpha_{0} \in \operatorname{Aut}\left(A^{(n)}(P)\right.$ such that $\overline{\alpha_{0}}=\alpha$ (see Lemma $(\overline{B .2}))$. By the hypothesis of induction there exists $t \in A^{(n)}(P)$ such that $\bar{x}=\bar{t}$. If $b \in\left[t, \alpha_{0}\right]$, then $b \in A^{(n+1)}(P)$ and $\bar{b}=\left[\bar{t}, \overline{\alpha_{0}}\right]=[\bar{x}, \alpha]=\bar{a}$. Similarly, we can see the converse. Now, we have $A^{(n)}(P) \subseteq \omega_{P}$ if and only if $A^{(n)}(\bar{P})=\{e\}$. This completes the proof.

(ii) This follows from (4.8) and (4.10).

Definition 4.11. Let $P$ be a polygroup, we define $k_{0}(P)=P$ and for every $n \in \mathbb{N}$,

$$
\begin{aligned}
k_{n}(P) & =\left\langle h \in[x, \alpha] \mid x \in k_{n-1}(P), \alpha \in \operatorname{Aut}(P)\right\rangle \\
& =\left\langle\left[k_{n-1}(P), \operatorname{Aut}(P)\right]\right\rangle .
\end{aligned}
$$

Polygroup $P$ is called an autonilpotent of class at most $n$ if $k_{n}(P) \subseteq \omega_{P}$. The smallest integer $n$ such that $k_{n}(P) \subseteq \omega_{P}$ is called the autonilpotency class. It is abvious that for every $n \in \mathbb{N}$, $e \in k_{n}(P)$ and $k_{n}(P) \leq_{c} P$ and hence a group $G$ is an autonilpotent group if and only if there exists some $n \in \mathbb{N}$ in such a way that $k_{n}(G)=\{e\}$. In a similar way one can see that if $A u t(P)=\{I\}$, then $P$ is autonilpotent.

Example 4.12. Let $P=\{e, a, b\}$ be the polygroup as in Example 4.9 . Then $k_{n}(P)=\{e, a, b\}$ and so $P$ is not autonilpotent.

Example 4.13. (i) Let $G=D_{8}=\left\{e, a, a^{2}, a^{3}, b, a b, a^{2} b, a^{3} b\right\}$ the dihedral group of order 8 and let $\{e, c\} \cong \mathbb{Z}_{2}$ and $P=\{e, c\}[G]$. Then

$$
\operatorname{Aut}(G)=\left\{\alpha_{i, j}: G \rightarrow G \mid \alpha_{i, j}(a)=a^{i}, \alpha_{i, j}(b)=a^{j} b, i \in\{1,3\}, j \in\{0,1,2,3\}\right\},
$$

and $\operatorname{Aut}(P) \cong \operatorname{Aut}(G)$, hence we get $k_{1}(P)=\langle a\rangle \cup\{c\}, k_{2}(P)=\left\langle a^{2}\right\rangle \cup\{c\}, k_{3}(P)=\{e, c\}$. Therefore, $P$ is autonilpotent.

(ii) Consider the polygroup $P$ in Example 4.9, since for all $n \geq 1, k_{n}(P)=\{1,2,6,7\}$ and $\omega_{P}=\{1,2\}$, we get that $P$ is not an autonilpotent polygroup.

Corollary 4.14. Let $P$ be a polygroup. Then

(i) $k_{n+1}(P) \subseteq k_{n}(P)$.

(ii) $k_{n}(P)=\left\langle\left[x, \alpha_{1}, \cdots, \alpha_{n}\right] \mid x \in P, \alpha_{1}, \cdots, \alpha_{n} \in \operatorname{Aut}(P)\right\rangle$. 
Proof. (i) By definition and induction, the proof is clear.

(ii) Using induction on $n$, we have

$$
\begin{aligned}
k_{n+1}(P) & =\left\langle\left[k_{n}(P), \operatorname{Aut}(P)\right]\right\rangle=\left\langle[x, \alpha] \mid x \in k_{n}(P), \alpha \in \operatorname{Aut}(P)\right\rangle \\
& =\left\langle\left[x, \alpha_{1}, \cdots, \alpha_{n}\right], \alpha\right]\left|x \in P, \alpha_{i}, \alpha \in \operatorname{Aut}(P), i=1, \cdots, n\right\rangle,
\end{aligned}
$$

then we get our claim.

Example 4.15. Let $P=\{1,2,3,4,5,6,7,8,9\}$ be the polygroup in Example 4.0 . It is easy to see that $k_{3}(P)=\{1,2\}$. Therefore, we conclude that $P$ is autonilpotent.

Theorem 4.16. Every autonipotent group $G$ is nilpotent.

Proof. It is easy to see that $l_{n}(G) \subseteq k_{n}(G), n \geq 0$ and this completes the proof.

Example 4.17. $S_{3}$ is not nilpotent and hence is not autonilpotent.

The following example, shows that the converse of the above theorem, is not necessarily true.

Example 4.18. Let $G=\mathbb{Z}_{6},\{\overline{0}, a\} \cong \mathbb{Z}_{2}$ and $P=\{\overline{0}, a\}[G]$. Then Aut $(P)=\left\{I,\left(\begin{array}{lll}\overline{1} & \overline{5}\end{array}\right)(\overline{2} \quad \overline{4})\right\}$ and for every $n \geq 1, k_{n}(P)=\{\overline{0}, \overline{2}, \overline{4}, a\}$ and hence $P$ is not autonilpotent but it is nilpotent.

Definition 4.19. Let $P$ be a polygroup. Define $Z_{0}(P)=\omega_{P}$, for every $n \in \mathbb{N}$,

$$
Z_{n}(P)=\left\langle x \in P \mid[x, \alpha] \subseteq Z_{n-1}(P), \forall \alpha \in \operatorname{Aut}(P)\right\rangle .
$$

Theorem 4.20. Let $P$ be a polygroup. Then

$$
\omega_{P}=Z_{0}(P) \subseteq Z_{1}(P) \subseteq \cdots \subseteq Z_{n}(P) \subseteq \cdots .
$$

Proof. Let $\alpha \in \operatorname{Aut}(P)$. Since $\alpha\left(\omega_{P}\right) \subseteq \omega_{P}$ (by Lemmas B.d and [3.2), we get

$$
Z_{0}(P)=\omega_{P} \subseteq\langle x \in P \mid \bar{x}=\bar{\alpha}(\bar{x}), \forall \alpha\rangle=Z_{1}(P),
$$

where $\bar{x}=\beta(x)$, and so by induction the proof is obtained.

Theorem 4.21. Let $P$ be a polygroup and $n \geq 0$. Then

$$
k_{n}(P) \subseteq \omega_{P} \quad \text { if and only if } Z_{n}(P)=P .
$$

Proof. Let $k_{n}(P) \subseteq \omega_{P}$. Then by induction on $i$, we show that $k_{n-i}(P) \subseteq Z_{i}(P)$. Let $x \in$ $k_{n-i-1}(P)$. Then for every $\alpha \in A u t(P),[x, \alpha] \subseteq k_{n-i}(P)$. By using the hypothesis of induction, we have $[x, \alpha] \subseteq Z_{i}(P)$ and so $x \in Z_{i+1}(P)$. Now for $i=n$ we obtain that $P=k_{0}(P) \subseteq Z_{n}(P) \subseteq P$, that is $Z_{n}(P)=P$.

Conversely, if $Z_{n}(P)=P$, then by induction we prove that $k_{i}(P) \subseteq Z_{n-i}(P), 0 \leq i \leq n$. Suppose that $h \in[x, \alpha]$, where $x \in k_{i}(P)$ and $\alpha \in A u t(P)$. Then by the hypothesis of induction we conclude that $x \in Z_{n-i}(P)$. Hence $h \in[x, \alpha] \subseteq Z_{n-i-1}(P)$. Letting $i=n$ implies that

$$
k_{n}(P) \subseteq Z_{0}(P)=\omega_{P} .
$$


Corollary 4.22. Let $P$ be a polygroup. Then $P$ is an autonilpotent polygroup if and only if there exists some $n \in \mathbb{N}$ in such a way that $Z_{n}(P)=P$.

Corollary 4.23. Every autonipotent polygroup is autosolvable.

Proof. Let $P$ be a polygroup. At first, we prove that $A^{(n)}(P)$ is characteristic in $P$. Let $\alpha, \alpha_{1} \in$ Aut $(P), x \in P$, then $\alpha\left(\left[x, \alpha_{1}\right]\right)=\alpha(x) \alpha \alpha_{1}\left(x^{-1}\right)=\left[\alpha(x), \alpha \alpha_{1} \alpha^{-1}\right] \subseteq A(P)$. Therefore, $A(P) \leq{ }_{c} P$, thus we conclude that $A^{(n)}(P) \leq_{c} P$, for all $n \in \mathbb{N}$. Now, by induction on $n$ we show that

$$
A^{(n)}(P) \subseteq k_{n}(P) \text {. }
$$

For $n=1$, we have $A^{(1)}(P)=A(P)=k_{1}(P)$. Now, suppose that $A^{(n)}(P) \subseteq k_{n}(P)$. Then

$$
\begin{aligned}
A^{(n+1)}(P) & =A\left(A^{(n)}(P)\right)=\left\langle\left[A^{(n)}(P), \operatorname{Aut}\left(A^{(n)}(P)\right]\right\rangle\right. \\
& \subseteq\left\langle\left[k_{n}(P), \operatorname{Aut}(P)\right]\right\rangle=k_{n+1}(P) .
\end{aligned}
$$

Now, suppose that $P$ is an autonilpotent polygroup. Then, there exists $n \in \mathbb{N}$ such that $k_{n}(P) \subseteq$ $\omega_{P}$ and so we obtain $A^{(n)}(P) \subseteq \omega_{P}$, and the proof is completed.

Theorem 4.24. Let $P_{1}$ and $P_{2}$ be two polygroups and $n \geq 1$. Then,

(i) $A^{(n)}\left(P_{1}\right) \times A^{(n)}\left(P_{2}\right) \subseteq A^{(n)}\left(P_{1} \times P_{2}\right)$,

(ii) If $P_{1} \times P_{2}$ is an autosolvable polygroup, then $P_{1}$ and $P_{2}$ are autosolvable.

Proof. (i) We prove our claim by induction on $n$. For $n=1$, it is obvious. Now, suppose that $\left(h_{1}, h_{2}\right) \in A^{(n+1)}\left(P_{1}\right) \times A^{(n+1)}\left(P_{2}\right)$. Then without loss generality suppose that $h_{i} \in\left[x_{i}, \alpha_{i}\right]$, for $i=1,2$, where $x_{i} \in A^{(n)}\left(P_{i}\right)$ and $\alpha_{i} \in \operatorname{Aut}\left(A^{(n)}\left(P_{i}\right)\right.$. By the hypothesis of induction we conclude that $\left(x_{1}, x_{2}\right) \in A^{(n)}\left(P_{1} \times P_{2}\right)$. Define $\alpha=\left(\alpha_{1}, \alpha_{2}\right)$ by $\alpha(x, y)=\left(\alpha_{1}(x), \alpha_{2}(y)\right)$. Clearly $\alpha \in \operatorname{Aut}\left(A^{(n)}\left(P_{1} \times P_{2}\right)\right)$. Hence, $\left(h_{1}, h_{2}\right) \in\left[\left(x_{1}, x_{2}\right),\left(\alpha_{1}, \alpha_{2}\right)\right] \subseteq A^{(n+1)}\left(P_{1} \times P_{2}\right)$.

(ii) Since $P_{1} \times P_{2}$ is an autosolvable polygroup, there exists $n \in \mathbb{N}$ such that

$$
A^{(n)}\left(P_{1}\right) \times A^{(n)}\left(P_{2}\right) \subseteq A^{(n)}\left(P_{1} \times P_{2}\right) \subseteq \omega_{P_{1} \times P_{2}}=\omega_{P_{1}} \times \omega_{P_{2}} .
$$

It follows that $A^{(n)}\left(P_{i}\right) \subseteq \omega_{P_{i}}$, for $i=1,2$. Hence $P_{1}$ and $P_{2}$ are autosolvable.

Example 4.25. Let $G=\mathbb{Z}_{2} \times \mathbb{Z}_{2}$. Then Aut $(G) \cong S_{3}$, Aut $(G)=G$ and $A\left(\mathbb{Z}_{2}\right)=\{\overline{0}\}$. Therefore, $G$ is not autosolvable while $\mathbb{Z}_{2}$ is autosolvable. On the other hand,

$$
\{(\overline{0}, \overline{0})\}=A\left(\mathbb{Z}_{2}\right) \times A\left(\mathbb{Z}_{2}\right) \subset A\left(\mathbb{Z}_{2} \times \mathbb{Z}_{2}\right) .
$$

The above example shows that Theorem 4.24 is not true in general.

Theorem 4.26. Let $K$ be a characteristic subpolygroup of a polygroup $P$. Then,

(i) $\operatorname{Aut}(K) \subseteq \operatorname{Aut}(P)$,

(ii) $A^{(n)}(K) \subseteq A^{(n)}(P)$,

(iii) If $P$ is autosolvable, then $K$ is autosolvable.

Proof. It is straightforward.

The following example shows that the condition in previous theorem is neccessary.

Example 4.27. Let $P$ be the polygroup in Example 4.3. We consider the non-characteristic subpolygroup $K=\{1,2,3,5,8\}$. It is easy to see that $A(K)=K$ and hence $K$ is not an autosolvable subpolygroup of autosolvable polygroup $P$. 


\section{Conclusions}

In this paper, we introduce the autosolvable and autonilpotent polygroups using the automorphisms and the following results are obtained from it:

(i) Relations $\beta, \gamma, \tau_{n}$ and $\nu_{n}$ are examples of invariant relations.

(ii) Using automorphism define autosolvable polygroups and prove that each autosolvable group is a solvable group.

(iii) With the concept of autocommutator, autonilpotent polygroup is introduced and it is proved that each autonilpotent polygroup is an autosolvable polygroup.

(iv) The symmetric group $\mathbb{S}_{3}$ is neither an autonilpotent group nor an autosolvable group but the dihedral group $D_{8}$ is both an autosolvable and autonilpotent group.

(v) With respect to the concept of autonilpotent polygroups, we investigated the relation between of autonilpotent groups and nilpotent groups.

(vi) It investigated the subpolygroup of autonilpotent polygroups are not necessarity autonilpotent polygroup.

\section{References}

[1] H. Aghabozorgi, B. Davvaz, M. Jafarpour, Nilpotent groups derived from hypergroups, Journal of Algebra, 383 (2013), 177-184.

[2] H. Aghabozorgi, M. Jafarpour, B. Davvaz, Solvable polygroups and derived subpolygroups, Communications in Algebra, 41 (2013), 3098-3107.

[3] P. Corsini, Prolegoment of hypergroup theory, Second Edition, Aviani Editor, 1993.

[4] B. Davvaz, Polygroup theory and related systems, World Scientific, 2013, Doi:10.1142/8593.

[5] D. Freni, A note on the core of a hypergroup and the transitive closure $\beta^{*}$ of $\beta$, Annali di Matematica Pura ed Applicata, 8 (1991), 153-156.

[6] D. Freni, A new characterization of the derived hypergroup via strongly regular equivalences, Communications in Algebra, 30 (2002), 3977-3989.

[7] A. Mosayebi Dorcheh, k-nilpotent groups based on hypergroup, Journal of Algebraic Hyperstructures and Logical Algebras, 2 (2021), 61-72.

[8] A. Mosayebi Dorcheh, M. Hamidi, R. Ameri, On auto-engel polygroups, Mathematics Interdisciplinary Research, 6 (2021), 63-83.

[9] F. Parvaneh, M.R.R. Moghaddam, Some properties of autosolvable groups, Journal of Mathematical Extension, 5(1) (2010), 13-19.

[10] T. Vougiouklis, Hyperstructures and their representions, Hadronic Press Inc, 1994. 